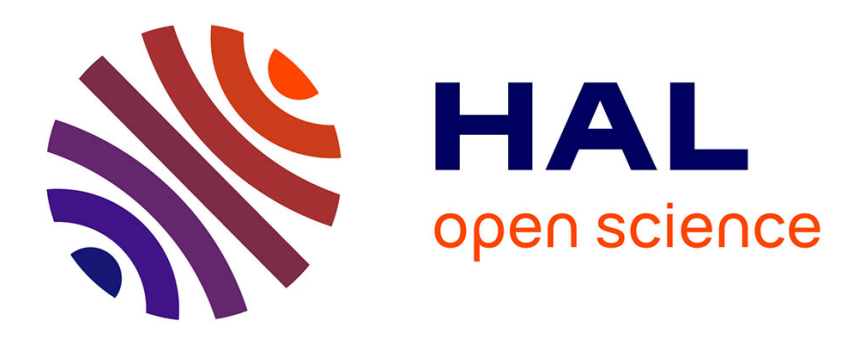

\title{
Noter le futur : témoignage et politique suivant Svetlana Alexievitch
}

Frédérik Detue

\section{To cite this version:}

Frédérik Detue. Noter le futur : témoignage et politique suivant Svetlana Alexievitch. Littérature, 2012, 168 (4), pp.85-102. 10.3917/litt.168.0085 . hal-02613967

\section{HAL Id: hal-02613967 \\ https://hal.science/hal-02613967}

Submitted on 8 Jun 2020

HAL is a multi-disciplinary open access archive for the deposit and dissemination of scientific research documents, whether they are published or not. The documents may come from teaching and research institutions in France or abroad, or from public or private research centers.
L'archive ouverte pluridisciplinaire HAL, est destinée au dépôt et à la diffusion de documents scientifiques de niveau recherche, publiés ou non, émanant des établissements d'enseignement et de recherche français ou étrangers, des laboratoires publics ou privés. 
FRÉDÉRIK DETUE

\section{NOTER LE FUTUR : TÉMOIGNAGE ET POLITIQUE SUIVANT SVETLANA ALEXIEVITCH}

J'étudierai ici l'œuvre de Svetlana Alexievitch intitulée La Supplication : Tchernobyl, chronique du monde après l'apocalypse, et parue en 1997 dans sa version originale russe Černobyl'skâa molitva (Hronika buduŝego), littéralement «La Prière de Tchernobyl (Chronique du futur) » ${ }^{1}$. Je voudrais poser la question de savoir s'il s'agit d'un témoignage. Car cela ne va pas de soi. Certes, l'événement dont traite La Supplication s'origine dans l'accident survenu le 26 avril 1986 à la centrale nucléaire de Tchernobyl, en Ukraine, et, selon l'édition de 1996 de la Belaruskaâ ènciklopediâ citée par l'auteur, c'est «la plus grande catastrophe technologique du $\mathrm{XX}^{\mathrm{e}}$ siècle» $(S, 7)$. Puis la démarche d'Alexievitch a été d'enquêter trois années durant dans son pays, la Biélorussie, qui est le plus touché par cette catastrophe ; elle a interrogé plus de cinq cents personnes, de sorte que son livre se présente bien comme un recueil de témoignages composé à partir de ces entretiens. Cependant, le témoignage comme art d'écrire s'est inventé au $\mathrm{XX}^{\mathrm{e}}$ siècle en lien avec les violences politiques de masse ; il est l'œuvre d'une victime, rescapée ou non, de la terreur moderne, qui dépose contre les coupables et autres responsables, qui relate son expérience collective pour la donner à connaître et à comprendre par-delà le secret et l'oubli, et qui la transmet aussi au nom de tous les disparus. On peut donc parler de témoignage quand une œuvre témoigne de crimes de guerre, d'un nettoyage ethnique, d'un génocide, de la détention en camp de concentration : Ceux de 14 de Genevoix, Au cour de l'enfer de Gradowski, Hiroshima, fleurs d'été de Hara ou Récits de la Kolyma de Chalamov font œuvre de témoignage. Comme la catastrophe de Tchernobyl ne procède pas d'une intention criminelle, on peut s'interroger sur le statut du livre d'Alexievitch.

Je soutiendrai néanmoins que ce livre s'inscrit dans la tradition de l'art testimonial, pour la raison que, malgré le caractère accidentel de la catastrophe, il vise essentiellement à faire comprendre un crime politique : un crime aux multiples facettes, qui réside d'abord et surtout dans la gestion de la catastrophe après coup. C'est pourquoi «[c]e livre ne parle pas de Tchernobyl, mais du monde de Tchernobyl» $(S, 30)$. Pour ce qui est des premières mesures, c'est un régime totalitaire qui est mis en cause, celui de l'Union soviétique. À cet

\footnotetext{
${ }^{1}$ Svetlana Alexievitch, La Supplication: Tchernobyl, chronique du monde après l'apocalypse, trad. du russe par Galia Ackerman et Pierre Lorrain, Paris, Éditions J'ai lu, coll. «Documents», 2006. Référence abrégée à ce livre, désormais : S. Version originale : Svetlana Aleksievič, Cinkovye malčiki. [Černobyl'skaâ molitva (Hronika buduŝego).] Moskva, Ėksto-Press, 2001. Référence abrégée à ce livre : ČM.
} 
égard, l'accusation de crime contre l'humanité ne semble pas abusive. Cependant, le monde de Tchernobyl existe pour bien longtemps encore, or, à aucun moment, il ne ressort de $L a$ Supplication que la catastrophe est mieux gérée depuis 1991 : c'est dire qu'en «not[ant] le futur » $(S, 33)$, Alexievitch interroge également la responsabilité de la Biélorussie actuelle et, plus largement, du monde postsoviétique tout entier.

\section{DÉNI TOTALITAIRE DE LA CATASTROPHE : TCHERNOBYL N'A PAS EU LIEU}

«Pour Tchernobyl, il faudra bien répondre un jour... [...] Comme pour 1937. Même si ce n'est que dans cinquante ans ! Même s'ils sont vieux ! Même s'ils sont morts ! Ce sont des criminels !» $(S, 211)$. Dans le livre d'Alexievitch, la voix de Vassili B. Nesterenko s'élève avec force colère et indignation contre la gestion de la catastrophe par les autorités soviétiques. Sans doute peut-on discuter le parallèle que le physicien établit avec la Grande terreur ; car, pour une question de mécanique politique, spécialement, l'analogie tourne court. Reste que, dans la suite de son réquisitoire, il dénonce de façon convaincante une dynamique totalitaire. Dès le jour de l'accident, il a éprouvé à ses dépens la culture soviétique du secret et du mensonge :

J'appelais sur une ligne gouvernementale, mais l'affaire était déjà strictement confidentielle. Dès que j'ai mentionné l'accident, la liaison a été coupée. Bien sûr, tout était écouté. Inutile de préciser par qui. Les organes concernés. L'État dans l'État. Et le fait que moi, le directeur de l'Institut de l'énergie nucléaire de l'Académie des sciences de Biélorussie, membre correspondant de l'Académie des sciences, je voulais parler au premier secrétaire du Comité central n'y changeait rien. Le secret s'étendait à moi aussi. $(S, 211$.

Quand, à force d'obstination, il parvient à joindre le premier secrétaire, Sliounkov, celui-ci ne veut rien entendre : «On m'a déjà fait un rapport [...]. Il y a bien eu un incendie, mais il a été maîtrisé » $(S, 212)$. V. B. Nesterenko rend compte ainsi de l'absorption de la société par l'État qui l'a empêché alors de lancer une campagne d'information et de «trouver des moyens d'agir » $(S, 215)$ : d'une part, il analyse que «[la] science [avait] été entraînée dans la politique » $(S, 212)$, et qu'en conséquence, quelle que fût sa légitimité scientifique, un physicien ne pouvait penser « donner des leçons au Comité central » $(S, 214)$; d'autre part, il propose une analyse du phénomène bureaucratique, dénonçant « $[\mathrm{u}] \mathrm{n}$ complot de l'ignorance et du corporatisme » $(S, 214)$ : «les responsables ne se faisaient pas du souci pour les gens, ils s'en faisaient pour leur pouvoir. Nous vivons dans un pays de pouvoir et non un pays d'êtres humains. L'État bénéficie d'une priorité absolue. Et la valeur de la vie humaine est réduite à zéro » $(S, 214-215)$. Le crime découle de la logique totalitaire suivante : d'abord, le parti est 
une instance au-dessus de tout, seule détentrice de la vérité ; puis la vérité qu'il délivre n'a d'autre fin que ses propres intérêts de pouvoir. Suivant cette logique, soit le réel peut servir les intérêts du parti, soit il est dénié et transformé de sorte qu'il s'accommode de la vérité du parti. Or le réel de Tchernobyl, qui ne pouvait servir les intérêts du parti, n'a pu être dénié et transformé qu'au prix d'un « sacrifice humain » $(S, 99)$.

Face à la catastrophe de Tchernobyl, l'incompréhension est bien compréhensible, audelà de toute considération politique ; j’y reviendrai, la démesure et le caractère inédit de l'événement justifient à eux seuls une sorte d'effet de sidération, y compris chez les spécialistes de la physique. Mais la réalité totalitaire détermine dès l'origine un certain type d'incompréhension. Dans l'idéal, l'homme nouveau soviétique est « un homme doté de la capacité de ne pas se laisser affecter, et donc surprendre par ce qui arrive » ${ }^{2}$, étant donné que la vérité du parti a toujours par avance défini pour lui ce qui est de l'ordre du possible et de l'impossible. Or la vérité du parti détermine que l'accident de Tchernobyl est impossible, et c'est pourquoi il est spécialement incompréhensible: tel est le ressort du «complot de l'ignorance ».

«Injonction [est] faite à chacun de ne pas penser ce qui contredit ou met en défaut les thèses officielles $»^{3}$, et Tchernobyl est donc impensable, puisque, « avant Tchernobyl l'atome était surnommé "le travailleur pacifique" » $(S, 202)$, que «les centrales nucléaires, c'était l'avenir » $(S, 204)$. Des témoins rappellent en effet qu'avant Tchernobyl, existait en Union soviétique un «culte de la physique » $(S, 179)$, que «[t]out le peuple soviétique a volé dans le cosmos avec Gagarine » $(S, 135)$ et que c'est ainsi toute une époque, celle de la conquête de l'espace, qui se définissait par la foi en l'atome. Tel était l'endoctrinement, dont se souvient un témoin : «Nos centrales nucléaires ne présentent aucun risque. On pourrait les construire sur la place Rouge. Elles sont plus sûres que des samovars » $(S, 95)$. Or, «élev[er] [tout un peuple] dans l'idée que l'atome pacifique soviétique n'[est] pas plus dangereux que le charbon ou la tourbe » $(S, 212)$, c'est incorporer une vérité officielle dans tout un édifice social de sorte qu'elle ne puisse être éliminée « sans risquer de ruiner tout l'édifice » ${ }^{4}$, et c'est en partie pourquoi l'accident de Tchernobyl a été perçu comme une menace sérieuse pour le régime; l'idée rétrospective que «ce n'est pas seulement Tchernobyl qui a explosé, mais le régime communiste » $(S, 247)$, ne doit pas être reçue comme une figure de rhétorique.

\footnotetext{
${ }^{2}$ Claude Lefort, La Complication : Retour sur le communisme, Fayard, 1999, p. 136.

${ }^{3}$ Ibid., p. 229.

${ }^{4}$ Hannah Arendt, Les Origines du totalitarisme : 3. Le Système totalitaire, trad. fr., Le Seuil, 1972, p. 89 : « Une fois que les slogans de [la] propagande sont intégrés dans une "organisation vivante" [expression empruntée à Hitler], ils ne peuvent être éliminés sans risquer de ruiner tout l'édifice ».
} 
Lors de l'accident, « [1]'idée que l'atome pacifique pouvait tuer n'entrait pas dans [les] esprits » $(S, 154)$, et il s'est donc agi qu'elle n'y entre pas, envers et contre la réalité. Quant à la stratégie mise en œuvre, là encore, le témoignage de V. B. Nesterenko se révèle précieux. «Pourquoi est-ce que les dosimétristes de votre Institut courent partout dans [Minsk] en semant la panique ? », lui a demandé Sliounkov $(S, 213)$; et lui de commenter : «Il fallait parler de physique, des lois de la physique. Et eux, ils [...] cherchaient des ennemis !» $(S$, 213-214). De nouveau dans l'histoire du régime soviétique - après les fictions staliniennes du complot trotskiste ou du complot des blouses blanches, par exemple -, on a eu recours à la fiction du complot de façon à justifier le besoin d'une contre-conspiration. Une véritable propagande de guerre s'est diffusée, dont beaucoup se souviennent : «À la télévision, les émissions se multipliaient [...] Et toujours le commentaire : Les Occidentaux sèment la panique en diffusant des calomnies délibérées au sujet de l'accident » $(S, 147)$; «Camarades, ne cédez pas aux provocations, tonnait la télé, jour et nuit » $(S, 223)$.

Or ces slogans impliquent aussi des directives, suivant le principe du «centralisme démocratique $»^{5}$. L'ordre de ne pas semer la panique s'est ainsi diffusé de haut en bas dans la société soviétique, grâce à l'amour de la discipline, de l'autorité, de l'ordre et de l'uniformité de communistes plus ou moins de bonne foi, tel Vladimir M. Ivanov, ancien premier secrétaire du comité du parti du district de Slavgorod :

On lit aujourd'hui dans les journaux : les communistes trompaient le peuple, lui cachaient la vérité. Mais nous avions notre devoir... Nous recevions des télégrammes du Comité central, du comité régional du parti... Notre mission était d'empêcher la panique. La panique, vraiment, est une chose terrible. Les gens étaient pendus aux nouvelles. [...] Et il y avait la peur, les rumeurs. C'était cela qui assommait la population, et non la radiation... [...] On ne peut pas dire que l'on dissimulait les choses volontairement. [...] On agissait en vertu de considérations politiques supérieures. $(S, 200$, trad. modifiée ; $\check{C} M, 407-408.)^{6}$

Du moment que le devoir d'obéir assurait de son bon droit, tous les moyens étaient bons pour «dissimuler le malheur » $(S, 146)$. D'abord, la rétention d'information était de rigueur. De façon symptomatique, «dans les premiers jours qui ont suivi la catastrophe, les livres sur les radiations, sur Hiroshima et Nagasaki et même sur la découverte de Röntgen ont disparu des bibliothèques » $(S, 92)$. Tandis que les mensonges se déversaient en «incroyable

\footnotetext{
${ }^{5}$ Lioudmila D. Polenskaïa décrit bien le fonctionnement centralisé du parti (absolument pas infléchi par l'adjectif « démocratique ») : «Dans notre district, nous attendions les instructions du comité régional qui lui attendait celles de Minsk. Bien sûr, à Minsk, on attendait celles de Moscou. C'était une très longue chaîne au bout de laquelle quelques personnes prenaient les décisions. Nous étions complètement sans défense. [...] Le sort de millions de personnes se trouvait entre les mains de quelques individus » $(S, 181-182)$.

${ }^{6}$ Pour une définition critique de l'homme nouveau soviétique, voir C. Lefort, La Complication, op. cit., p. 136137.
} 
quantité » $(S, 148)$, notamment dans les journaux ${ }^{7}$, « des montagnes de papiers avec la mention "strictement confidentiel" » ont exigé le secret, «sur l'accident », « sur les résultats du traitement des malades » et «sur le degré de contamination radioactive du personnel ayant participé à la liquidation » $(S, 167)$. Mais encore fallait-il que cette loi du silence fût respectée: beaucoup de témoignages rendent compte ainsi des méthodes d'intimidation employées par la police secrète. Le cas de V. B. Nesterenko, sur qui a pesé le spectre terroriste des années trente et qui a finalement « été victime d'un infarctus » $(S, 217)$, est peutêtre exceptionnel, son entêtement à vouloir dire la vérité étant lui-même exceptionnel ${ }^{8}$. Mais les « liquidateurs » ${ }^{9}$ ont eux aussi subi les pressions exercées par le parti. Pendant leur séjour à proximité de la centrale, on leur a ordonné de « ne pas entrer en contact avec la population » $(S, 147)$, et on les a surveillés de près s'ils s'avisaient d'écrire (voir $S, 161)$. Puis, avant leur départ définitif, ils ont été prévenus, généralement par « un collaborateur du K.G.B. » $(S, 80)$, «que les intérêts de l'État exigeaient le maintien du secret» $(S, 88)$, qu'il valait mieux «ne jamais raconter à personne ce [qu'ils avaient] vu» $(S, 80)$, et ils ont dû « signer un document de confidentialité » $(S, 86)$. On les a fouillés «pour qu'ils n'emportent pas de photos », témoigne la journaliste Irina Kisseleva, qui ajoute: «Le K.G.B. confisquait même les pellicules aux équipes de télévision. Ils les rendaient après les avoir exposées à la lumière. Que de documents ont été ainsi détruits !» $(S, 210)$.

«La seule chose que l'on pouvait filmer, c'étaient les actes d'héroïsme » $(S, 139)$, précise Sergueï V. Sobolev, et cela en dit long sur la version révisée de la catastrophe que l'on a présentée. Contre les prétendues provocations «antisoviétiques » des « ennemis » extérieurs (occidentaux) ou intérieurs, le pouvoir soviétique s'est voulu rassurant, soutenant, tel Gorbatchev, que « la situation [était] sous contrôle » et qu'il «n'y [avait] rien d'horrible » $(S$, 146). Dès lors, le mot d'ordre de la contre-conspiration a été que «nous devions vaincre » $(S$, 98). Le témoin cité ici, le liquidateur Arkadi Filine, exprime sa perplexité : «vaincre qui ? L'atome? La physique ? L'univers ?»; selon lui, ce mot d'ordre procède de la rationalité instrumentale dont procédait déjà la construction de la centrale de Tchernobyl, et qu'un autre

\footnotetext{
${ }^{7}$ «L'incroyable quantité de mensonges liés à Tchernobyl n'a pas d'équivalent, sauf pendant la guerre » $(S, 148)$, dit un liquidateur. Exemple spectaculaire : «Les journaux écrivaient : "Au-dessus du réacteur, l'air est pur". Nous avons ri, nous avons juré. L'air est peut-être pur, mais les doses énormes !» $(S, 187)$.

${ }^{8} \mathrm{~V}$. B. Nesterenko énumère toutes les brimades qu'il a endurées : «[son] Institut s'est vu confisquer - sans explication - tous les appareils destinés au contrôle des radiations », puis on a fait disparaître ses dossiers, et on l'a menacé d'《[exil] dans des contrées lointaines» $(S, 216)$, d'《intern[ement] en asile psychiatrique », d'« accident de voiture [organisé] » ou encore d'« information judiciaire pour activités antisoviétiques »voire «pour escroquerie » $(S, 217)$.

9 «Liquidateur » est le terme d'usage (likvidator, en russe) pour désigner les personnes, généralement des soldats ou des réservistes, appelés dans la zone de Tchernobyl pour limiter les dégâts causés par l'accident. J'emploierai désormais les mots « liquider », « liquidation » et « liquidateur » sans guillemets.
} 
témoin qualifie de «paganisme soviétique »: cette idée que l'homme est «le maître, la couronne de la création » et qu' « il [a] le droit de faire ce qu'il v[eut] de la planète » $(S, 175)$. Il ne s'agissait surtout pas d'admettre « [q]ue l'homme [fût] impuissant devant les lois de la physique » $(S, 154)$. Or on voit ici que, plus que la foi en l'atome, l'enjeu était « [la] foi en la victoire » $(S, 201)$, puisque, en Union soviétique, « la victoire n’est pas un événement, mais un processus » $(S, 98)$, que chaque événement doit pouvoir être considéré depuis « le point de vue de la victoire prolétarienne », comme un moment de sa réalisation. En pratique, cette victoire-processus s'incarnait dans «un système militaire [fait pour] fonctionn[er] parfaitement dans des circonstances extraordinaires » $(S, 82)$; et c'est ainsi qu'on a mobilisé la société soviétique sur la base de son «éducation militaire » $(S, 126)$, de sa «prépar[ation] en permanence à une guerre future $»(S, 184)^{10}$. «Les communistes, en avant. Telle était la situation » $(S, 82)$; on a rappelé des groupes de réservistes et « on [les] a fait partir sur-lechamp, en urgence. Comme au front» $(S, 190)$. Des liquidateurs sont allés «travail[ler] au réacteur. Comme dans une tranchée en première ligne » $(S, 184)$. Et les journaux ont commencé à titrer: "Tchernobyl, lieu d'exploit ", "Le réacteur est vaincu", "La vie continue" » $(S, 98)$.

\section{SACRIFICE HUMAIN 1 : ABANDON DES LIQUIDATEURS}

Une telle mobilisation de la société pose le problème de la servitude volontaire. Des témoins vont jusqu'à rejeter la faute sur le peuple soviétique : «Qui est coupable ? Personne, à part nous-mêmes ! » $(S, 126)$, exclame Nina K. Jarkova, et Zoïa D. Brouk de dénoncer dans le même sens le faux partage «peuple saint, gouvernement criminel» $(S, 169)^{11}$. La construction de l'homme nouveau soviétique ne se limitait pas au cadre du parti, en effet. Pour justifier sa soumission et celle de ses collègues, Marat P. Kokhanov, ingénieur en chef de l'Institut dirigé par V. B. Nesterenko, invoque «l'habitude de croire » d'une génération de l'après-guerre qui a «grandi dans la foi » $(S, 165)$; or il s'agissait moins de croyance que d'amour de la discipline et de l'uniformité, en réalité ; à la question : «Pourquoi avons-nous gardé le silence alors que nous savions ? », il répond : «Nous avons obéi sans un murmure parce qu'il y avait la discipline du parti, parce que nous étions communistes » $(S, 165)$. Certes, le parti appuyait son autorité sur un arsenal idéologique, lequel formait un ensemble de représentations et de valeurs (comme la foi en l'atome ou celle en la victoire) que la société

\footnotetext{
${ }^{10}$ Voir également $S, 138$ : «nous avons été élevés pour devenir des soldats. C’était tout le sens de notre éducation. Nous sommes mobilisés en permanence, toujours prêts à faire l'impossible. »

${ }_{11}^{1}$ ¿J'ai compris plus tard, quelques années plus tard, que nous avions tous participé... à un crime... à un complot... » $(S, 171)$, poursuit-elle.
} 
soviétique était censée défendre comme un seul homme. Mais l'idéologie, en Union soviétique, visait moins la constitution de véritables convictions qu'une capture de la pensée telle que «la distinction entre fait et fiction (i.e. la réalité de l'expérience) et la distinction entre vrai et faux (i.e. les normes de la pensée) n'existent plus $»^{12}$. C'est pourquoi l'habitude de croire était avant tout l'habitude de ne pas penser par soi-même - quitte à devoir «[se] ment[ir] à [soi]-même » $(S, 141)$-, et d'« attendre qu'on nous dise les choses, qu'on nous les annonce » $(S, 183)$. On doit alors reconnaître « un mode de structuration des rapports sociaux tel que, pour reprendre une formule de Soljenitsyne, le peuple en arrive à devenir son propre ennemi $\gg^{13}$, parce que, par un mécanisme d'identification, la société intériorisait la vérité officielle, même quand celle-ci procédait d'une logique criminelle; mais on doit dans le même temps discuter l'idée qu'il soit « difficile de dire qui était coupable, dans le système qui existait alors » $(S, 107)$, comme si «[1]es ordres [qui] arrivaient d'en haut » n'émanaient pas de dirigeants dont on pouvait déterminer les responsabilités, selon leur pouvoir de décision. La réflexion à mener à présent sur ce qu'il en a été de la prévention et de la protection de la population ne peut que renforcer cette analyse : manque de rationalité des autorités, force d'inertie de la bureaucratie, absence de politique de santé publique, arbitraire, corruption, autoritarisme, cynisme... - les chefs d'accusation sont légion, dans La Supplication.

Quand le sens du devoir et l'amour du nous induisent l'homme nouveau soviétique à sacrifier sa vie sans se poser de questions - parce que « [le] Soviétique est incapable de penser exclusivement à lui-même, à sa propre vie » $(S, 191)$, parce que, « quand il faut y aller, il faut y aller ! La patrie nous a appelés !» $(S, 158)$-, la servitude volontaire prend la forme, effrayante, d'un folie suicidaire collective, et il faut y réfléchir à deux fois, avant de parler d'héroïsme à la façon de la propagande soviétique. C'est l'avis d'A. Filine : «En toute honnêteté, je n'ai pas vu de héros, là-bas. Tout juste des fous qui n'en avaient rien à foutre, de leur vie » $(S, 95)$. Certes, il a fallu empêcher à tout prix qu'une explosion nucléaire succédât à l'explosion thermique du 26 avril $^{14}$ puis que le réacteur «s'enfon[çât] dans les eaux

\footnotetext{
${ }^{12}$ H. Arendt, Le Système totalitaire, op. cit., p. 224.

${ }^{13}$ C. Lefort, La Complication, op. cit., p. 227.

${ }^{14}$ Voir $S, 137-138$ : «À un moment donné, il existait un risque d'explosion nucléaire. Pour l'éviter, il a fallu vider le réservoir d'eau lourde sous le réacteur, pour qu'il ne s'écroule pas dedans. L'eau lourde est une composante du combustible nucléaire. Vous imaginez ce qui aurait pu se passer. La mission était donc de plonger dans l'eau lourde et d'ouvrir la soupape de vidange. À celui qui y parviendrait, on a promis une voiture, un appartement, une datcha et une pension à ses proches jusqu'à la fin de leurs jours. Et il y a eu des volontaires. Les gars ont plongé à plusieurs reprises et ils sont parvenus à ouvrir la soupape. On a donné sept mille roubles à l'ensemble de l'équipe et l'on a oublié les voitures, les appartements et le reste. Mais ce n'est pas à cause de cela qu'ils ont plongé ! [...] Ces gens ne sont plus de ce monde... Il ne reste que des documents dans notre musée... Des noms... Mais s'ils avaient refusé de le faire ?» Voir également J.-P. Dupuy, Retour de Tchernobyl, Le Seuil, 2006, p. 80-81. En cas d'explosion nucléaire, les effets de la catastrophe auraient été démultipliés de façon
} 
souterraines », et il a fallu aussi déverser des tonnes de sable «dans l'orifice brûlant de la centrale » $(S, 140)$ et élever le fameux « sarcophage »-cette «pyramide du $\mathrm{XX}^{\mathrm{e}}$ siècle » $(S$, 86). Ces tâches-là de liquidation de l'accident, tout aussi nécessaires et urgentes que dangereuses, justifient peut-être en partie qu'on ait planifié des pertes en vies humaines, comme le soutient S. V. Sobolev ${ }^{15}$. Il paraît raisonnable, cependant, d'entendre ce témoin, qui avertissait $\mathrm{S}$. Alexievitch : « Ne mentionnez pas trop les miracles de l'héroïsme soviétique. Bien sûr, il y en a eu quelques-uns. Mais avant tout, ce que l'on voyait, c'était l'incurie et le désordre $\gg(S, 80)$.

Qu'ils renient ou non leur premier « élan héroïque », qu'ils reconnaissent ou non qu'il « était insinué » $(S, 76$, trad. modifiée ; $\check{C} M, 306)$, les liquidateurs qui survivent témoignent en majorité de ce qu'il leur a coûté. Un pilote d'hélicoptère, ancien membre du parti, parle de son « enthousiasme d'homme » de l'époque, de la honte faite et des sanctions prises contre deux autres navigateurs qui ont refusé de survoler le réacteur, mais de ce que, «après neuf opérations et deux infarctus, [il est] d'un avis différent » $(S, 87)$. «Il ne fallait pas envoyer une telle quantité de gens. Les soumettre à l'irradiation. Il fallait des spécialistes et non pas seulement du matériel humain » $(S, 88)$, juge-t-il rétrospectivement. Beaucoup font part, dans ce sens, de ce qu'ils sont arrivés aux abords de la centrale sans rien connaître des risques auxquels ils s'exposaient, et qu'en l'absence de toute prévention, ils ont commis des imprudences et même des folies souvent sans qu'il y ait besoin de les contraindre ; «ce n'était qu'en passant que nous nous demandions pourquoi nous n'avions pas de dosimètres, pourquoi on ne nous donnait pas de comprimés par prophylaxie, pourquoi nous n'avions pas de machines à laver pour nettoyer nos vêtements de travail tous les jours et non deux fois par mois », note Victor Latoun, photographe $(S, 191)$. Quand les liquidateurs voulaient s'informer, de toute façon, on était «incapable de [leur] répondre », et on les rappelait à l'ordre : «Qu'avez-vous besoin de savoir ? Exécutez les ordres » $(S, 190)$. Ils avaient été au mieux «prépar[és] à une guerre nucléaire, mais pas à Tchernobyl » $(S, 170)$; là, rien ne servait de savoir comment éviter une onde de choc. Il aurait mieux valu « appren[dre] [...] à expulser des radionucléides de l'organisme » $(S, 127)$, mais cela supposait de savoir « que la contamination radioactive du terrain était le facteur qui affectait le plus l'organisme », ce que «personne ne [leur] avait dit » $(S, 157)$. Sur place, on comprenait assez vite, malgré tout, que la poussière «n'était pas de la poussière toute simple, qu'elle était radioactive » $(S, 114)$,

exponentielle, souligne l'auteur : «la ville de Kiev aurait disparu de la carte, la Biélorussie [aurait été] rendue impropre à la vie à jamais et l'Europe [...] [serait devenue] inhabitable pour une durée indéterminée $» \ldots$

${ }^{15}$ Voir $S, 140$ : «Aux réunions de la commission gouvernementale, on rapportait les choses d'une manière très simple : "Pour cela, il faut mettre une vie. Et pour ceci, deux ou trois vies..." » 
mais, même quand on distribuait des masques à gaz ou des respirateurs, «personne ne les portait » $(S, 79)$; « [i]l faisait $35^{\circ}$ à l'ombre : si tu les mets, tu crèves » $(S, 158)$, explique l'ingénieur chimiste Ivan N. Jmykhov. Les premiers jours, «[on les mettait] dès qu'une voiture passait en soulevant la poussière », mais «[d]eux mois plus tard, [on se] comport[ait] normalement» $(S, 185)$, rapporte pour sa part Alexandre Koudriaguine, «invalide au deuxième degré ». Aller sur le toit du réacteur n'a jamais cessé de faire peur, tout de même, mais il n'était pas question de refuser ; « [1] es volontaires iront sur le toit, et les autres, chez le procureur » $(S, 188)$, menaçait-on les récalcitrants. Or, quand « on [les] jetait comme du sable sur le réacteur» $(S, 81)$, là où «les radiations étaient telles que tous les équipements brûlaient » $(S, 88)$, les liquidateurs n'étaient certes plus comme les pompiers de la première heure «en chemise » $(S, 11)$, mais, même avec masques, combinaisons et gants, ils étaient beaucoup trop exposés : «On leur donnait des tabliers de plomb, mais la radiation venait d'en bas et, là, ils n'étaient pas protégés. Ils portaient des bottes ordinaires en similicuir... $(S$, 136-137), précise S. V. Sobolev.

I. N. Jmykhov note qu'à un moment, certains ont reçu des dosimètres, mais que ceuxci «n'étaient pas chargés »; «[e]n d'autres termes, on nous avait donné des joujoux, juste pour faire bien. Une sorte de psychothérapie » $(S, 162)$. Quant au soldat qui «a osé demander [à un général], devant toute l'unité : "Pourquoi nous dissimule-t-on les mesures de radiation? Quelles doses recevons-nous ?" [...] le commandant de l'unité [l']a convoqué pour lui passer un savon: "C'est de la provocation! Tu sèmes la panique !" » $(S, 79)$. En fait, comme le déplore un liquidateur que, de peur, sa femme a ensuite quitté, «la quantité de radiations [qu'ils avaient] reçue demeurait "strictement confidentielle" ». « Même au moment de mon départ, ils ne m'ont pas dit combien j'en avais reçu. Les chiens !» $(S, 85)$, enrage-t-il, or il semble qu'on puisse généraliser ce constat. Ceux qui ont eu accès à leur fiche individuelle n'ont pas été plus avancés, car, même strictement confidentielles, les doses de radiation mentionnées étaient fictives ${ }^{16}$. Le liquidateur qui enrage de n'avoir rien su interroge : « Et comment nous soignerait-on? Nous n'avons pas rapporté le moindre document $\gg(S, 85)$. Or, comme le soulignent plusieurs anciens combattants de la guerre d'Afghanistan, le problème

\footnotetext{
16 « À la fin, le chiffre inscrit sur nos livrets militaires était le même pour tous : la dose moyenne de radiation multipliée par le nombre de jours. Cette dose était celle des tentes où nous habitions » $(S, 162)$, rapporte I. N. Jmykhov. Son témoignage est confirmé par celui d'un pilote d'hélicoptère : « [dans le centre du district, à Tchernobyl,] un dosimétriste mesurait le fond de la radiation. Or, nous nous trouvions tout de même à dix ou quinze kilomètres de la centrale. Ce chiffre était ensuite multiplié par le temps que nous avions volé dans la journée. Mais dans notre travail, nous survolions régulièrement le réacteur à bord de nos hélicoptères. Et, à la centrale, la radioactivité variait : un jour quatre-vingt röntgens, le lendemain, cent vingt... » $(S, 77)$.
} 
est bien que, contrairement à une guerre, où la «perspective de la mort est immédiate et non dans un avenir quelconque » $(S, 127)$, Tchernobyl « ne tuerait qu'après [leur] départ » $(S, 80)$.

\section{SACRIFICE HUMAIN 2 : UN PEUPLE ET UN MONDE À PART}

On a ainsi laissé livrés à eux-mêmes des centaines de milliers de liquidateurs qui, après avoir passé parfois jusqu'à « six mois » $(S, 83)$ à proximité de la centrale de Tchernobyl, «[ont disparu] dans les étendues infinies de [leur] grande patrie » $(S, 137)^{17}$. Mais il ne faudrait pas oublier le reste de la population à la façon de la propagande soviétique. V. B. Nesterenko préconisait deux mesures sanitaires d'urgence, le 26 avril 1986 : «traiter préventivement à l'iode toute la population [biélorusse] [...] évacuer les gens et le bétail dans un rayon de cent kilomètres » $(S, 211)$. Pour cause d'inertie bureaucratique, le premier ordre du physicien n'a été suivi d'aucun effet; il aurait été pourtant possible, sans faire peur aux gens, d' «introdui[re] des préparations à l'iode dans les réservoirs d'eau potable, en les ajoutant dans le lait », mais, à Minsk, « sept cents kilogrammes de ces préparations [...] sont rest[és] dans les entrepôts » $(S, 215)$. Quant à l'évacuation, elle a bien eu lieu, mais on a pris une décision insuffisante, en créant autour de la centrale une zone interdite d'un rayon de trente kilomètres, et non de cent ${ }^{18}$. Ainsi, depuis l'accident, toute une population vit dans des territoires contaminés. En 1996, dans la Belaruskaâ ènciklopediâ, on estime que cela concerne « un [Biélorusse] sur cinq », soit «2,1 millions de personnes, dont sept cent mille enfants » $(S, 8)$. Or, sur le long terme, c'est sans doute dans cette décision que réside le crime le plus grave : celui qui fait que, pour des millions d'habitants «privés de référence à l'accident comme étant ce qui arrive », «[1]'événement, c'est d'abord la vie quotidienne et le fait d'être brutalement plongé dans un monde doté de nouvelles règles, de nouveaux interdits », et par conséquent « [une] nouvelle condition humaine $»^{19}$.

\footnotetext{
${ }^{17}$ Dans ses Notes de Hiroshima, Kenzaburô Ôé consacre quelques pages marquantes aux cent trente-cinq hibakusha originaires d'Okinawa : «Après avoir subi les bombardements de Hiroshima ou de Nagasaki, ils sont revenus dans leur île natale. Comme s'ils s'exilaient avec leurs blessures sur quelque îlot perdu, où l'on ne savait rien des soins à apporter au syndrome des atomisés » (K. Ôé, Notes de Hiroshima, trad. fr., Gallimard, 1996, p. 28). Il évoque plus loin «le problème des soins à apporter aux personnes dispersées aux quatre coins du pays » (id., p. 66). Ce problème de l'accès à des soins spécialisés se pose pour quelque six cent mille liquidateurs de façon analogue - mais à l'échelle de l'URSS...

${ }^{18} \mathrm{~V}$. B. Nesterenko suggère que cela aurait pu être pire, car les autorités soviétiques ont fait une concession à l'opinion publique internationale : «Si nous étions restés dans un système fermé, derrière le rideau de fer, les gens seraient demeurés à proximité immédiate de la centrale. On y aurait créé une région secrète, comme à Kychtym ou Semipalatinsk... » $(S, 214)$.

${ }^{19}$ G. Grandazzi, "Tchernobyl, 20 ans après : l'avenir d'une catastrophe », Cahiers de la Chaire de responsabilité sociale et de développement durable (ESG, UQÀM), nº 2, avril 2006, p. 8.
} 
J'aborde ici ce qui fait de la catastrophe de Tchernobyl un événement sans équivalent - et donc pas comparable, entre autres, au bombardement d'Hiroshima. Le journaliste Anatoli Chimanski exprime cela :

\begin{abstract}
J'ai lu des ouvrages sur Hiroshima et Nagasaki [...]. C'est horrible mais compréhensible : une guerre atomique, le rayon de l'explosion... Tout cela, je peux me le représenter. Mais ce qui s'est passé ici n'entre pas dans ma conscience. [...] Je sens qu'une chose totalement inconnue de moi détruit tout mon monde antérieur, rampe, se glisse à l'intérieur de moi-même. J'ai discuté avec un scientifique : «Il y en a pour des milliers d'années, m'expliquait-il. La désintégration de l'uranium, il y en a pour un milliard d'années. Et pour le thorium, quatorze milliards. » $(S, 130$.
\end{abstract}

Très souvent, dans La Supplication, on juge ainsi que ce qui s'est passé à Tchernobyl « défie l'entendement » $(S, 135)$, que «[1]a compréhension philosophique de Tchernobyl est encore devant nous » $(S, 223)$, peut-être «dans vingt ou trente ans » $(S, 78)$; que « nous ne savons pas tirer les leçons de cette horreur [...] car il est impossible de l'appliquer à notre expérience humaine ou à notre temps humain » $(S, 94)$. C'est pourquoi le témoignage prend ici la forme de l'enquête. De façon à rendre son expérience des camps imaginable, Antelme s'est refusé à la traiter «comme un tout, donné une fois pour toutes, allant de soi, éloquent, à lui seul », mais, entre elle et lui, «[a] interpos[é] toute la grille d'une découverte, d'une mémoire, d'une conscience allant jusqu'au bout $»^{20}$. D'une façon analogue, «Tchernobyl [étant] un mystère qu'il nous faut encore élucider » $(S, 30)$, Alexievitch n'a cessé d'interroger ce mystère, en quête «trois années durant» $\mathrm{d}$ ' «un homme bouleversé [...] qui aurait été confronté à cela, face à face, et se serait mis à réfléchir » $(S, 32)$.

Le constat suivant lequel les milliers de tonnes de radionucléides envoyés dans l'atmosphère et retombés massivement sur la terre biélorusse équivalent «à trois cent cinquante bombes de Hiroshima » $(S, 213)$ est impressionnant. Cependant, l'éclair d'Hiroshima a provoqué instantanément la destruction d'une ville entière et quelque cent mille morts, tandis que la «lueur framboise, flamboyante » de Tchernobyl a offert un «très beau » spectacle aux témoins oculaires $(S, 153)$ et n'a tué, dans les premiers jours, qu'une dizaine de personnes, pompiers ou opérateurs de la centrale. «[L]es gens ne conçoivent pas d'autres images de l'apocalypse: explosions, incendies, cadavres, panique » (S, 197); comment comprendre alors que «la mort [puisse] être aussi belle » $(S, 154)$, puis qu'il y ait une telle «différence d'échelle entre ce qui s'[est] passé et le nombre de victimes [des premiers jours] » $(S, 118)$ ?

\footnotetext{
${ }^{20}$ G. Perec, « Robert Antelme ou la vérité de la littérature », in L.G. : Une aventure des années soixante, Le Seuil, 1992, p. 95.
} 
La nécessité de l'évacuation donne à penser la différence de nature qui distingue Tchernobyl d'Hiroshima. Tandis qu'on a pu reconstruire la ville japonaise jusqu'à effacer la mémoire de la catastrophe ${ }^{21}$, «[1]'homme s'en [est] allé pour toujours de [toute une région autour de Tchernobyl] » $(S, 193)$, devenue inhabitable. «Personne ne croyait que nous ne reviendrions plus. Un cas pareil ne s'était jamais présenté » $(S, 109)$, témoigne Katia P., évacuée de Pripiat. Mais Pripiat, où vivaient 50000 personnes, est devenue une «ville fantôme » $(S, 156)$ comparée parfois à Pompéi ${ }^{22}$, et en tout la Biélorussie a «perd[u] 485 [villages] », dont «soixante-dix [...] sont enterrés pour toujours » $(S, 7-8)$. La zone interdite de Tchernobyl est devenue une vaste «terre des morts», dans laquelle les liquidateurs ont fait œuvre de fossoyeurs, enterrant, outre les villages, « le bétail abattu » $(S$, $84)$, les chats et les chiens errants abattus ${ }^{23}$, «la couche contaminée de la terre » $(S, 158)$ ou encore « la "forêt rousse" abattue sur cent cinquante hectares autour du réacteur » $(S, 141)$, emplissant au total quelque «huit cents "sépulcres"» $(S, 141)$ - en fait de simples «fosses communes » $(S, 84)^{24}$. Cependant, s'il fallait bien délimiter cette zone, le fait qu'à seulement trente kilomètres de la centrale, «d'un côté de la route, il [soit] interdit de passer et [que], de l'autre, les vaches paiss[ent], les moissonneuses-batteuses travaill[ent] et les camions transport[ent] les céréales » $(S, 76)$ pose un très grave problème humain.

À propos de son expérience de liquidateur dans la zone interdite, A. Filine convoque « une parabole sur Lazare qui a regardé derrière le trait de l'interdit. Après cela [dit-il], il est devenu étranger parmi les siens, même si Jésus l'a ressuscité... » $(S, 97)$. Cette parabole vaut pour tout «le peuple de Tchernobyl » $(S, 194)$. À cet égard, le sort des malheureux pompiers de Pripiat appelés à éteindre l'incendie la nuit de l'accident est programmatique ; c'est pourquoi, à la suite de l'« Information historique », La Supplication s'ouvre sur le témoignage de la veuve de l'un d'entre eux. Comme les autres, atteint du syndrome aigu d'irradiation, celui-ci a été immédiatement mis en quarantaine puis transporté dans un «établissement

\footnotetext{
${ }^{21}$ Voir G. Anders, L'Homme sur le pont : Journal d'Hiroshima et de Nagasaki (1958), trad. fr., in Hiroshima est partout, Le Seuil, 2008, p. 141-142. À K. Ôé, il semble que « sur cette terre tout le monde, sans exception, tente d'effacer complètement de sa mémoire Hiroshima et l'absolue tragédie qui s'est produite en ce lieu » (Notes de Hiroshima, p. 134); or G. Anders reconnaît cet effacement dans la reconstruction d'Hiroshima, où plus rien n'évoque la catastrophe : dans ce sens, souligne-t-il, «la reconstruction est véritablement la destruction de la destruction, et du même coup le sommet de la destruction ».

22 Voir F. Lemarchand, «Petites mythologies de Tchernobyl», http://vivretchernobyl.blogspot.com/2008/06/frdrick-lemarchand-petites-mythologies.html (consulté le 17.07.2009).

${ }^{23} \mathrm{Au}$ moment de l'évacuation, il était interdit d'emporter les animaux domestiques, dont les poils étaient trop radioactifs (voir par exemple $S$, 45). Les liquidateurs recevaient l'ordre de «tirer systématiquement sur [les chats et les chiens errants] » $(S, 75)$ demeurés dans la zone, semble-t-il « pour éviter des épidémies » $(S, 101)$.

${ }^{24}$ « Nous soulevions la terre et l'enroulions comme un tapis », dit I. N. Jmykhov : «Un travail de fous. On ne peut quand même pas éplucher toute la terre, ôter tout ce qui est vivant... Si nous ne nous étions pas soûlés à mort toutes les nuits, je doute que nous eussions pu supporter cela. » $(S, 159)$.
} 
radiologique spécial » $(S, 15)$ à Moscou, où il est mort dans les deux semaines qui ont suivi ; « ce n'est plus votre mari, l'homme aimé, qui se trouve devant vous, mais un objet radioactif avec un fort coefficient de contamination » $(S, 22)$, avait-on prévenu sa femme, or cette condition humaine très spéciale a valu également des enterrements très spéciaux à ses collègues et lui : «Dans des cercueils en zinc, scellés, sous des dalles de béton » $(S, 25)$.

Les personnes évacuées, qui ont été exposées à des doses de radiation énormes - «de plusieurs dizaines de milliers de fois supérieures à celles des soldats qui gardent les zones d'essais nucléaires » $(S, 207)$-, témoignent de ce qu'eux, «les hibakush[a] de Tchernobyl» $(S, 111)$, «[ont] tous été transformés en curiosités ambulantes » $(\mathrm{S}, 44)$ et sont « devenus un peuple à part » $(S, 154)$, dont on craignait la contagion ; une mère rapporte qu'à l'école, les enfants «avaient peur de [son fils] et l'appelaient "la luciole" » $(S, 155)$. Or ce sentiment désespéré d'être « des étrangers. Des lépreux » $(S, 194)$ est partagé par les habitants des zones non évacuées bien que fort contaminées. Dans l'histoire du $\mathrm{XX}^{\mathrm{e}}$ siècle, jusque-là, on avait «expériment[é] des mutations de la nature humaine ${ }^{25}$ spécialement dans les camps; le «musulman »d'Auschwitz ou le «crevard» de la Kolyma, à la frontière de l'humain, en sont des figures de victimes exemplaires, dans les témoignages de Levi et de Chalamov. Le «monde de Tchernobyl » $(S, 30)$, qui n'est pas comparable aux camps, est cependant le terrain d'une nouvelle expérimentation, dont la victime porte cette fois - péjorativement - le nom de «Tchernobylien» $(S, 84)$ et où il s'agit aussi plus de survivre que de vivre. N. P. Jarkov parle du monde de Tchernobyl comme d' « un gigantesque laboratoire du diable » où l'« [o]n vient de partout » pour «prépar[er] l'avenir » mais où donc plus de deux millions de Biélorusses deviennent « des cobayes pour des expériences scientifiques [internationales] » $(S, 128),-$ « comme des "boîtes noires", les enregistreurs de vol des avions... Des "hommesboîtes"» $(S, 150)$, suivant un autre témoin.

Dès l'année qui a suivi l'accident, « [1]'impensable s'est produit : les gens se sont mis à vivre comme avant » $(S, 125)$; dix ans après, encore, «[1]es champs ont beau être parsemés de panneaux "Haute radiation", ils sont labourés... Les tractoristes travaillent dans des cabines ouvertes [...] et respirent de la poussière radioactive... » $(S, 224)$. Pourtant, rien, en vérité, n'est comme avant, et la question se pose pour beaucoup de savoir « comment vivre maintenant » $(S, 127)$, alors qu' « avec Tchernobyl, l'homme a levé la main sur tout » $(S, 121)$, que «Tchernobyl est désormais tout le temps avec nous » $(S, 124)$ et qu' « [i]l n'y aura plus

\footnotetext{
${ }^{25}$ H. Arendt, Le Système totalitaire, op. cit., p. 200 : «[le] dessein des idéologies totalitaires n'est [...] pas de transformer le monde extérieur, ni d'opérer une transmutation révolutionnaire de la société, mais de transformer la nature humaine elle-même ».
} 
jamais d'autre monde » $(S, 143)$. Car, sans que l'on puisse en éprouver la sensation ni s'en protéger, on « respir[e] de la radiation, [on] mang[e] de la radiation»; «tout est empoisonné » $(S, 127)$, mais de façon si imperceptible que «nous pouvons boire du thé autour d'une table, parler et rire sans nous apercevoir que la guerre a commencé » $(S, 220)$. C'est ainsi tout le monde de Tchernobyl qui apparaît comme lazaréen, du fait de la radiation :

On ne l'entend pas, on ne la voit pas, elle n'a ni odeur ni couleur, mais nous change physiquement et psychologiquement. Notre formule sanguine change, notre code génétique change, le paysage change... Quoi que nous pensions, quoi que nous fassions... Je me lève le matin, je bois le thé, je vais aux répétitions, je rencontre mes élèves... Et cela est suspendu au-dessus de moi. Comme un signe. Comme une question. Je ne peux comparer cela à rien. $(S, 197$.

Posséder un dosimètre, dans ces conditions, devient franchement angoissant : « Je lave le linge, chez moi. Il est si blanc, mais le dosimètre sonne [dit une femme]. Je prépare un gâteau, il sonne. Je fais le lit, il sonne. À quoi bon l'avoir ? Je donne à manger aux enfants et je pleure » $(S, 149)$. Les autorités dénoncent ce défaitisme, arguant que c'est justement la peur de la radiation, et non la radiation elle-même, qui provoque les maladies. N. K. Jarkova interroge, cependant : «On dit: "Vous êtes malades parce que vous avez peur. À cause de la [...] phobie de la radiation.” Mais pourquoi les petits enfants sont-ils malades ? $(S, 124)$. Cette femme angoissée par son dosimètre peut-elle faire autrement, tandis que ses enfants «passent leur temps à l'hôpital », que son aîné, «chauve » $(S, 149)$, est atteint d'une leucémie ? Il faut sans doute entendre ici le «cri » d'Arkadi P. Bogdankevitch, assistant médecin, qui « compulse chaque jour » les fiches médicales d'enfants nés entre 1985 et 1987 : «On prétend que ce n'est pas possible. Comment peuvent-ils vivre avec une thyroïde pareille ?» $(S, 122)$. Les enfants à qui N. K. Jarkova enseigne vivent mal, assurément : «Lorsqu'on les met en rang, s'ils restent debout quinze ou vingt minutes, ils s'évanouissent, saignent du nez. On ne peut ni les étonner ni les rendre heureux. Ils sont toujours somnolents, fatigués. Ils sont pâles, et même gris » $(S, 123)$. Le monde de Tchernobyl est ainsi un monde où les enfants « vont continuellement à des enterrements » $(S, 123)$, « pensent à la mort » $(S$, 196), «se demandent si cela fait peur ou non » $(S, 123)$ et où les petites filles jouent «à l'hôpital » $(S, 90)$ plutôt qu' «à l'école » et font mourir leurs poupées. Elles expliquent : «Parce que ce sont nos enfants et que nos enfants ne vont pas vivre. Ils vont naître et mourir » $(S, 151)$.

Dans un tel monde où la mort est omniprésente, la question de savoir « comment vivre maintenant» tend à se confondre avec celle de savoir «comment on meurt après Tchernobyl » $(S, 241)$. Mourir du mal aigu des rayons ou du « [cancer] de Tchernobyl, encore 
plus terrifiant [que le cancer ordinaire] » $(S, 244)$, fait peur: les employés d'une morgue jugent que «[1]es Tchernobyliens meurent de la façon la plus horrible » $(S, 247)$. Un liquidateur, qui a vu un ami devenir «énorme, comme un tonneau », et un voisin, opérateur d'une grue, devenir «noir comme du charbon » puis « rétréc[ir] jusqu'à la taille d'un enfant », confie : «Je n'ai pas peur de la mort en elle-même, mais je ne sais pas comment je vais mourir. [...] La seule chose que je sache avec certitude, c'est que ma vie ne sera pas longue, avec ce que j'ai » $(S, 86)^{26}$. Or ce sentiment que la mort est dans le temps, que l'on est en sursis, est partagé par tout le peuple de Tchernobyl. C'est ce qu'exprime la blague de Tchernobyl la plus courte : «Ils formaient un bon peuple, les Biélorusses !» $(S, 210)$. Comme la mort dans le temps n'a fait que commencer et « va durer [...] plusieurs générations » $(S$, 153), qu'elle est non seulement dans l'air, dans la terre et dans l'eau génération après génération, mais aussi dans les corps d'une génération à l'autre, la question de savoir comment on va vivre et mourir concerne aussi les générations futures, de sorte que l'on craint de donner la vie. «Nous avons peur [...] pour nos enfants... Pour les petits-enfants que nous n'avons pas encore » $(S, 195)$, dit Nadejda A. Bourakova; des amis à elle ont donné naissance à un enfant qui «a une énorme fente en guise de bouche et pas d'oreilles ». Comme ses copines de classe, sa fille adolescente s'inquiète : «Maman, si j'accouche d'un bébé difforme, je l'aimerai quand même » $(S, 194)$. Cette peur d'enfanter - à cause de possibles « séquelles génétiques » $(S, 217)$ - est devenue la norme, dans les territoires contaminés où l'on continue de vivre ; «Cela fait bien longtemps que je ne vois plus de femmes enceintes, heureuses... Des mères heureuses... » $(S, 144)$, observe une sage-femme. L'historien Alexandre Revalski, qui ignorait comme tant d'autres qu' «il ne fallait pas s'aimer [là-bas] » $(S, 90)^{27}$, rapporte ainsi qu'«en 1993 les femmes de Biélorussie ont subi, à elles seules, deux cent mille avortements [et que] la cause principale en est Tchernobyl $\gg(S, 172)^{28}$.

\footnotetext{
${ }^{26}$ Dans le monde concentrationnaire, où la mort est omniprésente, Jean Améry soutient qu' « on ne se préoccup[e] plus guère de savoir si ou bien que l'on d[oit] mourir, mais uniquement comment la chose se passer[a] », de sorte que la réalité du camp induit selon lui « l'effondrement total de la représentation esthétique de la mort » (Jean Améry, Par-delà le crime et le châtiment, trad. fr., Actes Sud, 2005, p. 50-51).

${ }_{27}$ « Tchernobyl se trouve dans ma propre maison [témoigne-t-il]. Il est dans l'être le plus cher pour moi, dans mon fils qui est né au printemps $1987 .$. Il est malade. Les animaux, même les cafards, savent à quel moment il convient d'enfanter. Les hommes ne le peuvent pas. » $(S, 172$.

${ }^{28}$ Estimation corroborée par la Belaruskâ̂ ènciklopediâ : «Les radiations constituent la principale source de déficit démographique. Dans les régions de Gomel et de Moguilev (qui ont le plus souffert de la tragédie), la mortalité est supérieure de $20 \%$ à la natalité $»(S, 8)$.
} 


\section{DÉNI POST-TOTALITAIRE DE LA CATASTROPHE : LOBBY NUCLÉAIRE INTERNATIONAL ET RISQUE DE « GLOBOCIDE »}

Telle est la singularité du monde de Tchernobyl, mais il y a encore une chose à déplorer : c'est que cette tragédie humaine donne lieu à une nouvelle forme historique de révisionnisme, voire de négationnisme. Logiquement, à partir du moment où l'on dit aux gens qu'ils sont malades parce qu'ils ont peur, on n'entend pas les reconnaître comme victimes. Le combat de Larissa Z., une mère, est exemplaire, à cet égard. « À la naissance, [sa fillette,] ce n'était pas un bébé, mais un sac fermé de tous les côtés, sans aucune fente [...] pas de foufoune, pas de derrière et un seul rein » $(S, 89)$. Or même une pathologie aussi complexe à la naissance peut être « considér[ée] [...] comme [une] malad[ie] habituel[le] ». Les médecins se justifiaient : «Dans vingt ou trente ans, lorsque l'on aura accumulé suffisamment de données sur Tchernobyl, on établira un lien entre ces maladies et les radiations ionisantes. Mais, pour l'instant, la médecine et la science ne disposent pas d'assez d'éléments » $(S, 91$ 92). Ainsi, pendant quatre ans, on a parlé de «malformations [...] congénitales » et refusé à cette mère «un certificat qui confirmait le lien entre des petites doses de radiation et [1]a terrible maladie [de sa fille] » $(S, 91)$; un fonctionnaire l'a même accusée de vouloir « des privilèges en tant que victime de Tchernobyl ». Mais elle «devai[t] savoir que ce n'était pas [leur] faute [à son mari et à elle] » $(S, 92)$; elle a donc lutté « contre les médecins, contre les fonctionnaires » quatre ans durant, et fini par obtenir gain de cause.

Cependant, pour une victime reconnue tant bien que mal, combien de victimes ignorées? Tandis que Kofi Annan, alors secrétaire général des Nations unies, évoquait en 2000 les «9 millions de victimes de Tchernobyl», le Forum Tchernobyl, organisme regroupant huit organisations internationales dépendant de l'ONU, osait affirmer en 2005 que «jusqu'à 4000 personnes au total pourraient à terme décéder des suites d'une radioexposition consécutive à l'accident [de Tchernobyl] $»^{29}$. Alexievitch n'a pas souhaité entrer dans ce débat sur les chiffres; mais les témoignages qu'elle recueille donnent l'idée de l'étendue du mensonge perpétré à l'échelle internationale. Car le rapport du Forum Tchernobyl en 2005 véhicule le même discours déjà commenté sur les méfaits de la peur, soutenant que «[1]es populations concernées ont été en vérité très gravement affectées, mais [...] parce qu'elle croyaient avoir été très gravement affectées ${ }^{30}$; que ce sont donc essentiellement des mécanismes physiologiques et psychologiques (liés au stress et à l'angoisse) qui sont en cause, dans les problèmes sanitaires qui ont fait suite à l'accident.

\footnotetext{
${ }^{29}$ Voir J.-P. Dupuy, Retour de Tchernobyl, op. cit., p. 51-52.

${ }^{30}$ Ibid., p. 56.
} 
Or la question que l'on peut se poser, face à un tel discours, concerne l'héritage totalitaire de ce que l'on a coutume d'appeler la «communauté internationale ». Car «[1]e silence criminel et l'effacement des traces entretenus par le lobby nucléaire international - en tout premier lieu, l'AIEA [1'Agence internationale pour l'énergie atomique] - face à la catastrophe de Tchernobyl, contribuent à nier l'humanité de l'homme de Tchernobyl » ${ }^{31}$ aussi clairement que pouvait le faire la politique des autorités soviétiques. C'est sans doute contre ce lobby nucléaire international que se joue l'actualité la plus vive de La Supplication; contre le discours indécent d'experts pour qui les Biélorusses feraient mieux de «se considérer comme d'heureux survivants $»^{32}$, qu'Alexievitch donne la parole aux Biélorusses - aux vaincus.

Le fait qu'en 2005, le prix Nobel de la paix ait été décerné à l'AIEA et à sa mission « d'accélérer et d'accroître la contribution de l'énergie atomique à la paix, à la santé et à la prospérité dans le monde entier $»^{33}$ en dit long sur le travail qu'il reste à accomplir pour recevoir cette parole des vaincus. Dans la quatrième de ses «Dix thèses pour Tchernobyl » intitulée «Distinguer un usage guerrier et un usage pacifique de l'énergie nucléaire est fou et mensonger », Günther Anders pose le problème de la conscience d'aujourd'hui :

Aujourd'hui, après Tchernobyl, dans la mesure où plus personne ne peut jouer les ignorants, ses avocats en sont venus à commettre consciemment un crime. Ce crime ne s'appelle pas seulement « génocide»-quel emploi de l'adverbe «seulement »! - mais «globocide», destruction du globe. Les partisans de l'énergie nucléaire mais aussi et surtout ceux des usines de retraitement de déchets et des surrégénérateurs ne sont en rien meilleurs que l'a été le Président Truman qui a fait bombarder Hiroshima. Ils sont même pires que lui, car les gens en savent aujourd'hui bien plus que le naïf président pouvait en savoir à son époque. ${ }^{34}$

Peut-être la foi soviétique en l'atome procédait-elle d'une vraie naïveté et le discours officiel suivant lequel les centrales nucléaires sont «plus sûres que des samovars » était-il sincère. C'est pourquoi je n'ai pensé pouvoir parler de crime qu'à propos de la gestion criminelle de la catastrophe. Mais une des leçons de Tchernobyl est pourtant que «quelques personnes se sont révélées capables de nous [des millions de personnes en Biélorussie] assassiner [qui] n'étaient ni des maniaques ni des criminels [mais] [d]e simples opérateurs de service dans une centrale nucléaire » $(S, 182)$. Comment dès lors juger que la foi en l'atome des experts internationaux

\footnotetext{
${ }^{31}$ G. Grandazzi, F. Lemarchand, V. Symaniec, « Témoigner sur Tchernobyl : les sciences humaines et l'art face à la catastrophe », in F. Dépelteau, A. Lacassagne (dir.), Le Bélarus : L'État de l'exception, P.U. Laval, 2003, p. 365.

${ }^{32}$ Voir J.-P. Dupuy, Retour de Tchernobyl, op. cit., p. 58.

${ }^{33}$ Ibid., p. 54.

34 G. Anders, «Dix thèses pour Tchernobyl » [juin 1986], trad. fr, in Écologie \& politique : Destination Tchernobyl, 2006, $\mathrm{n}^{\circ} 32$, p. 172-173. Je souligne.
} 
d'aujourd'hui est innocente - qui plus est, alors qu'elle prête son concours à la gestion criminelle de la catastrophe?

Sur la crainte que nous devrions avoir du «globocide» et des «meurtriers sans méchanceté » qui menacent de le commettre, la parole des vaincus nous éclaire, dans $L a$ Supplication, témoignant d'une prise de conscience étonnamment spontanée. Alors qu' «avant [Tchernobyl], [il] ne voy[ait] même pas le monde autour de [lui] », parce que, avant, c'était « comme si quelqu'un nous l'avait donné pour toujours » $(S, 126$, trad. modifiée ; $\breve{C} M, 344)$, le peuple de Tchernobyl lui porte une attention comme jamais il ne l'avait fait. Beaucoup partagent le sentiment de cet homme qui affirme que « les oiseaux, les arbres, les fourmis sont plus proches de [lui] qu'auparavant» $(S, 73)$. A. Chimanski n'a «jamais regard[é] [les fourmis] d'aussi près » que pendant l'évacuation de la zone : «Nous allons disparaître et elles ne s'en rendront même pas compte » $(S, 130)$, se fait-il la réflexion. L'opérateur de cinéma Sergueï Gourine projette quant à lui de «faire un film, et [de] tout voir au travers des yeux d'un animal » $(S, 121)$; or, si cela lui importe plus que de faire un film sur la Tchétchénie, c'est bien que Tchernobyl l'a projeté dans un temps de la fin. «Nous avons été privés de l'immortalité : voilà ce qui nous est arrivé » $(S, 198)$, dit Lilia M. Kouzmenkova. Si nous ne voulons pas devenir des contemporains de la fin des temps, ce mot de la fin mérite sans doute d'être entendu. 